\section{RevistadePolítica Económica y Desarrollo Sostenible}

Centro Internacional de Política Económica para el Desarrollo Sostenible
Revista de Política Económica y Desarrollo Sostenible

EISSN: 2215-4167 • Vol. 6 (1) • Julio-Diciembre, 2020: 1-24

DOI: https://doi.org/10.15359/peds.6-1.1

URL: http://www.revistas.una.ac.cr/politicaeconomica

Revista electrónica semestral publicada por el Centro Internacional de Política Económica para el Desarrollo Sostenible

Universidad Nacional, Lagunilla, Heredia Apartado 2393-3000

\title{
Los problemas actuales del desarrollo y la vigencia del pensamiento de Rodrigo Facio Brenes ${ }^{1}$
}

\section{Current problems on the development and relevance of the thought of Rodrigo Facio Brenes}

Francisco Esquivel Villegas ${ }^{2}$

francisco@franciscoesquivel.com

Fecha de recibido: 15 de julio de 2020. Fecha de corregido: 18 de agosto del 2020.

Fecha de publicación: 11 de noviembre del 2020

\section{Resumen}

El desarrollo de las economías abiertas necesita la complementariedad del mercado externo con la dinámica interna del aparato productivo. La expansión económica de Costa Rica desde mediados del siglo XIX hasta la década del 30 del siglo anterior reveló las limitaciones de una estrategia que se basó en el predominio del crecimiento exportador, sin establecer los mecanismos apropiados para el estímulo del mercado interno. El análisis de Rodrigo Facio Brenes, elaborado a inicios de la década del 40 del siglo anterior, dio aportes significativos a la definición de una estrategia alternativa que se puso en práctica gracias a un período de transformaciones políticas de naturaleza progresista. Al respecto, el crecimiento de Costa Rica desde mediados del siglo anterior hasta la década del 70 mostró una superación del modelo exportador tradicional de naturaleza mono exportador. La crisis mundial de la década del 70 produjo un estancamiento productivo y una inflación elevada en los principales mercados internacionales, afectando el funcionamiento de la estrategia alternativa aplicada por Costa Rica. Esto condujo a una nueva etapa exportadora desde finales de la década del 80. Sin embargo; la estrategia que orientó esa nueva etapa deterioró la complementariedad del proceso exportador con la dinámica interna de la economía. Se produjo un crecimiento importante, pero sin generar los estímulos apropiados para el mercado interno. En consecuencia, es necesaria una revisión de la estrategia exportadora para que se restablezca la interrelación adecuada entre lo externo y lo interno de la economía. Por esto, las definiciones estratégicas de Facio siguen vigentes, aunque el contexto operativo sea diferente al de la época que le tocó vivir.

Palabras claves: Competitividad, Encadenamientos productivos, Estrategia de desarrollo; Macroeconomía; Teoría económica.

1 Este artículo respondió a una convocatoria sobre el tema "Pensamiento y práctica de la Política Económica", en el marco del 25 aniversario del CINPE-UNA

2 Economista costarricense, con más de cuatro décadas de práctica profesional. Actualmente se dedica a la consultoría internacional y a la docencia de posgrado. 
Revista de Política Económica y Desarrollo Sostenible • EISSN: 2215-4167

Vol. 6 (1) • Julio-Diciembre, 2020: 1-24

DOI: https://doi.org/10.15359/peds.6-1.1

Esquivel

URL: http://www.revistas.una.ac.cr/politicaeconomica

\begin{abstract}
The development of open economies needs the complementarity of the external market with the internal dynamics of the productive apparatus. The economic expansion of Costa Rica from the mid-nineteenth century to the 1930s revealed the limitations of a strategy that was based on the predominance of export growth without establishing the appropriate mechanisms to stimulate the domestic market. The analysis of Rodrigo Facio Brenes, formulated at the beginning of the 1940s, made significant contributions to the definition of an alternative strategy that was put into practice thanks to a period of progressive political transformation. In this regard, the growth of Costa Rica from the mid-twentieth century to the 1970s surpassed the traditional mono-product export model. The global crisis of the 1970s produced productive stagnation and high inflation in the major international markets, affecting the operation of the alternative strategy applied by Costa Rica. This led to a new exporting stage commencing in the late 1980s. However, the strategy that guided this new stage deteriorated the complementarity of the export process with the internal dynamics of the economy. Significant growth occurred, without however generating the appropriate stimulus for the internal market. Accordingly, a review of the export strategy is necessary to restore the appropriate interrelationship between the external and internal sectors of the economy. For this reason, the strategic definitions of Facio continue to be relevant, although the operational context is different from that of the time he lived through.
\end{abstract}

Keywords: Competitiveness, Production chains, Development strategy, Macroeconomics, Economic theory.

\title{
Introducción
}

Rodrigo Facio Brenes fue una de las figuras más influyentes en la definición de la estrategia económica que aplicó Costa Rica en la segunda mitad del siglo anterior. Facio formuló una crítica profunda de la estrategia que orientó el desarrollo económico del país por aproximadamente cien años, hasta mediados del siglo XX. Su agudo diagnóstico le permitió comprender con gran claridad la debilidad del "crecimiento hacia afuera", sin vínculo con la dinámica interna económica y social. Por eso formuló una propuesta de desarrollo que combinaba el "crecimiento hacia afuera" con el "crecimiento hacia adentro". Gracias a la dinámica política que vivió Costa Rica a mediados del siglo pasado, este pensamiento influyó en la estrategia de desarrollo que adoptó el país.

Para Facio:

El rezago de Costa Rica durante el período "cafetalero" radicaba en la dependencia de un sector exportador limitado, que generaba una excesiva inestabilidad: "el monocultivo,... entraña permanentemente (una) amenazadora posibilidad: que una disminución en el volumen de la producción, una baja de sus precios o un desarreglo en el mecanismo de su tráfico internacional, produce ineludiblemente, una crisis de entidad nacional, pues no existen otras ramas económicas suficientemente fuertes e independientes (Facio, 1972, pp.101).

Además, la falta de un mercado interno sólido se relacionaba con la exclusión:

Mientrasla densidad denuestra poblacióny sus necesidadeshan crecidoininterrumpidamente, la producción nacional de artículos indispensables de consumo inmediato, no ha seguido 
Revista de Política Económica y Desarrollo Sostenible • EISSN: 2215-4167

Vol. 6 (1) • Julio-Diciembre, 2020: X-XX

DOI: https://doi.org/10.15359/peds.6-1.1

Esquivel

URL: http://www.revistas.una.ac.cr/politicaeconomica

el mismo ascenso... (Esto) importa además, en el plano puramente humano, el gravísimo fenómeno del infra consumo popular permanente... El campesino, sobre todo, vive sumamente mal (Facio, 1972, pp.110).

Ante esto, Facio consideraba que se debía aplicar una nueva estrategia de desarrollo que incluyera la expansión del mercado interno, aumentando y diversificando la producción nacional. Así, el café perdería importancia en la estructura productiva, disminuyendo el papel de los ciclos externos. No obstante; el cambio de la organización productiva no significaba marginar al café. Además de impulsar el mercado interno, se debía fortalecer estratégicamente a la exportación, acorde con las necesidades del desarrollo nacional. No se trataba de eliminar el café, sino de ponerlo a funcionar de manera articulada al proceso de expansión del mercado local.

Por otra parte, la propuesta asignaba un papel a la inversión extranjera, de acuerdo con las necesidades de la expansión productiva nacional. No se trataba de rechazar el capital extranjero, sino de aprovecharlo como una palanca más de la nueva organización productiva. Es decir; se concebía el ingreso de la inversión externa como un factor positivo, siempre que apoyara la nueva estrategia económica.

La estadística económica confirma lo que generó la estrategia: durante las décadas del 50 y del 60 , la producción para el mercado interno creció un $250 \%$, mientras que la producción para exportación aumentó más del $300 \%$. ¡El "crecimiento hacia afuera” se combinó con el "crecimiento hacia adentro"!

El análisis económico de Rodrigo Facio, y su influencia en la toma de decisiones de su época, dan base para considerarlo el economista del siglo XX en el país. Su reflexión sigue válida hoy, en agosto de 2020, ya que, en los últimos 30 años, se ha generado una estrategia económica que ha producido una diversificación exportadora, impulsando el crecimiento productivo; pero, simultáneamente, se mezcla con la exclusión y la reproducción crónica del desempleo y la pobreza.

A inicios de 2020, aún antes de la aparición de la pandemia por COVID-19, había una quinta parte de la población activa, desempleada o subempleada, lo que se relaciona con una seria dificultad para impulsar encadenamientos productivos y con una política pública sin horizonte, tardía e ineficiente. La pandemia, desde luego, agudizó el problema; pero, como expresión coyuntural. Es fundamental comprender que el desempleo, como desequilibrio estructural, en su mayor parte ha sido producto de la insuficiencia del modelo exportador en mención, a lo largo de tres décadas. Además, la desigualdad se profundiza constantemente, de acuerdo con el índice de Gini que no muestra techo todavía. Es decir; el "crecimiento hacia afuera" no se ha articulado adecuadamente con el "crecimiento hacia adentro". Por lo tanto, la reflexión de Facio sigue teniendo vigencia.

Los contenidos que se presentan a continuación están basados en una parte de un documento anterior del autor (Esquivel, 2015, pp. 27 - 60, 119 - 138). El análisis y los contenidos estadísticos se han actualizado en esta presentación. 
Revista de Política Económica y Desarrollo Sostenible • EISSN: 2215-4167

Vol. 6 (1) • Julio-Diciembre, 2020: 1-24

DOI: https://doi.org/10.15359/peds.6-1.1

Esquivel

URL: http://www.revistas.una.ac.cr/politicaeconomica

\section{1.- La crítica de Facio al modelo mono exportador y su propuesta de desarrollo}

En Costa Rica y en Latinoamérica la estrategia exportadora del siglo XIX y de inicios del siglo XX experimentó fuertes críticas luego de la crisis económica mundial iniciada en 1929. La caída de los ingresos externos, derivada de la enorme contracción de los mercados de las economías más avanzadas, generó un impacto devastador en las economías atrasadas como las latinoamericanas. En ese sentido, una contracción de las exportaciones significaba un golpe al principal bastión de la estructura productiva como lo era la producción agrícola de exportación. Tal efecto se propagaba sobre el resto de la estructura productiva, que estaba organizada alrededor de dicho baluarte. Desde el punto de vista de la demanda agregada, la reducción de las exportaciones se tradujo en una disminución de los ingresos de la economía en su conjunto, lo que limitó la dinámica del mercado interno, completamente dependiente del impulso exportador. Además, la reducción de los ingresos externos suponía una fuerte caída de la capacidad de compra de productos importados.

La Comisión Económica para América Latina y el Caribe (CEPAL) lideró la crítica al modelo exportador latinoamericano de inicios del siglo XX. Raúl Prebisch, fue su conductor, desde la década del 50, luego de dirigir el Banco Central de Argentina, en el período posterior a la crisis mundial iniciada en 1929. Fue acompañado por otras figuras del pensamiento económico latinoamericano, como el brasileño Celso Furtado, el chileno Aníbal Pinto Santa Cruz y el mexicano Juan Francisco Noyola, entre otros.

La CEPAL consideró, en esa época, que la expansión exportadora no aportaba los elementos suficientes para que las economías de la región se pudieran desarrollar. La experiencia latinoamericana de la década del 30, ante la crisis mundial, generó suficientes hechos que alimentaron la reflexión cepalina. De esta manera, la Institución propuso un modelo económico alternativo basado en el impulso del mercado interno, como opción de desarrollo, el llamado "desarrollo hacia adentro".

El análisis comprobó que las economías grandes de la región (Brasil, Argentina y México) experimentaron un crecimiento de la producción local que abastecía al mercado interno, ante la dificultad para comprar productos importados (derivada de la limitación de los recursos generados por la exportación). Tal expansión productiva se había producido por iniciativa del sector privado, sin ninguna política nacional que la estimulara. En consecuencia, se promovió el establecimiento de una política estatal que impulsara ese proceso mediante diversos estímulos que estaban al alcance de las instancias gubernamentales. Se trataba de promover una política de sustitución de importaciones. Una parte de lo que antes se compraba en el mercado externo sería producido mediante la capacidad productiva local: se sustituiría el producto importado que atendía la demanda interna por producto local. El crecimiento resultante aportaría una oportunidad de recuperar la senda de expansión del empleo y del ingreso, en un marco de equilibrio externo (porque reducía la dependencia de las fluctuaciones del mercado externo y de las compras de productos importados).

En Costa Rica, durante la década del 40, también se produjo una reflexión sobre estrategia de desarrollo, en un entorno propicio para los cambios de la organización económica y social. 
En ese momento se estableció una reforma social, que cambió la dinámica política que vivió el país en las décadas anteriores. Esto sucedió en el contexto de los movimientos sociales del campo (principalmente del sector bananero) y de la ciudad que presionaban por opciones de desarrollo efectivas. En la misma dirección la Iglesia se comprometía con la causa de amplios sectores de la población y participaba de los procesos que impulsaban los cambios. Además, surgieron grupos intelectuales que apostaban por el cambio social en el marco de un crecimiento sostenido que no dependiera del viejo modelo oligárquico-transnacional.

El intelectual que aportó más a la discusión de la estrategia de desarrollo, en esa época, fue Rodrigo Facio. Formuló una crítica contundente del modelo oligárquico-transnacional con el fin de proponer una alternativa de desarrollo para la Costa Rica de su momento. La reflexión comenzaba con una valoración de los efectos limitantes que producía el monocultivo: "Nuestra producción cafetalera no está, pues, regida por ningún criterio de economía nacional: ella se lleva a cabo exclusivamente para satisfacer los mercados internacionales”. (Facio, 1972, pp.99)

[...] el monocultivo, por serlo, entraña permanentemente (una) amenazadora posibilidad: que una disminución en el volumen de la producción, una baja de sus precios o un desarreglo en el mecanismo de su tráfico internacional, produce ineludiblemente, una crisis de entidad nacional, pues no existen otras ramas económicas suficientemente fuertes e independientes, ni aun de mercado interno, capaces de compensar o equilibrar la situación anormal sobrevenida (Facio, 1972, pp.101)

De acuerdo con el análisis de Facio, la expansión cafetalera se produjo en el marco de una división internacional del trabajo que no permitía superar el rezago del país:

El fenómeno monocultista del café,... es profundamente antieconómico desde el punto de vista nacional, y no como se sostiene por algunos, que es consecuencia, no solo natural, sino saludable, de la división internacional del trabajo, según la cual, obedeciendo a razones geográficas, económicas y de otra índole, la producción de cada país debe especializarse hasta el último grado. Así, los mantenedores de tal tesis, sostienen que Costa Rica debe estimular y proteger la producción del café, sin preocuparse por la producción de otros artículos, ya que por la calidad del grano y su renombre en el mercado mundial, le resulta más productivo adquirir esos otros artículos en el exterior con el oro importado por éste, que dedicarse a producirlos aquí directamente. De modo que hasta propugnarían, si fuese el caso, inundar de cafetales todo el territorio nacional y comprar con el oro que teóricamente esa producción nos reportara todas las cosas que necesitamos para vivir" (Facio, 1972, pp.110).

En este contexto, el predominio de la producción cafetalera producía una reducción relativa del mercado interno, generando el desabastecimiento de los productos básicos, necesarios para mejorar el nivel de vida de la población (Facio, 1972): 
Revista de Política Económica y Desarrollo Sostenible • EISSN: 2215-4167

Vol. 6 (1) • Julio-Diciembre, 2020: 1-24

DOI: https://doi.org/10.15359/peds.6-1.1

Esquivel

URL: http://www.revistas.una.ac.cr/politicaeconomica

Mientrasladensidaddenuestrapoblacióny sus necesidadeshan crecidoininterrumpidamente, la producción nacional de artículos indispensables de consumo inmediato, no ha seguido el mismo ascenso - se ha estancado en algunos casos, ha conservado el mismo volumen en otros, no ha tenido en los más un crecimiento acorde con el de la demanda - obligándonos a comprarle al extranjero en cantidades crecientes, mucho de lo que necesitamos para vivir (pp.119).

[...] Pero este problema..., importa además, en el plano puramente humano, el gravísimo fenómeno del infra consumo popular permanente... El problema irresuelto de la deficiencia que soporta nuestro pueblo en sus consumos básicos de alimento, vestuario, medicinas y habitación (pp. 123).

[...] El campesino, sobre todo, vive sumamente mal: ... el factor esencial de la situación... es de índole simplemente económica, y estriba en la carestía de los productos... (porque hay) una desorganización anti-nacional y anti-democrática de la agricultura patria." (pp. 124)

Se observa, de esta manera, una visión nítida de Facio sobre la estrecha relación entre el desarrollo del mercado interno y la aplicación de una efectiva política social. Lo económico y lo social estaban claramente integrados en su enfoque, por lo que los cambios que se proponían debían ser aplicados en diferentes dimensiones de la realidad nacional.

Además, la organización productiva del modelo cafetalero se basaba en una estructura de propiedad concentrada:

Mientras el $75,59 \%$ de los propietarios cafetaleros posee entre 1 y 2.000 arbustos, el $0,86 \%$ de ellos, o sean ciento sesenta y uno, poseen arriba de 50.000 arbustos cada uno, y el $0,07 \%$, o sean siete propietarios, poseen más de 400.000 cada uno de ellos: o en otras palabras, que el fundamento de la primera industria agrícola del país [...]

la constituye la gran propiedad [...] Sabemos muy bien que la industria cafetalera sólo es en realidad remunerativa para quienes producen en grandes cantidades y para quienes realizan la transformación industrial del fruto, y que esta última operación está a cargo, en forma monopolística, de los propietarios de beneficios, que por lo demás, lo son, en general, los grandes terratenientes [...]. El pequeño propietario,... no viene a ser en realidad, sino un peón privilegiado del beneficiador, expuesto permanentemente, por su condición de dependencia, a la pérdida de su propiedad. (Facio, 1972, pp. 104).

Dada esta realidad, Facio consideraba que se debía aplicar una nueva estrategia de desarrollo basada en la expansión del mercado interno, sin olvidar el papel de la actividad 
cafetalera y de la inversión extranjera. Tal estrategia estaría orientada por un "criterio económico costarricense", es decir; la estrategia siempre pensaría en lograr lo mejor para la expansión de la producción nacional, en beneficio de los sectores amplios de la población:

De acuerdo con un estudio objetivo de las necesidades nacionales - y es lo que atrás hemos pretendido hacer - la finalidad de una política económica para el interior, debe ser el aumento y la diversificación de la producción nacional: así se atenuaría la preeminencia del café y con ello nuestra dependencia del exterior y todos los otros perjuicios del monocultivo (Facio, 1972, pp. 169).

También, la expansión productiva tendría un perfil social. Como se expresó, el enfoque de Facio tenía claridad de la relación estrecha entre la dimensión económica y la dimensión social. Para que fuera factible la reforma social, que avanzaba en el plano político, era necesario una propuesta de desarrollo del mercado interno basada en la producción local:

Y no será posible sin la adopción de un plan de diversificación y aumento de la producción nacional, solucionar la desnutrición popular costarricense; y no lo será, porque sólo ese plan podrá voluminizar y popularizar la riqueza nacional, y el problema de desnutrición es simple cuestión de miseria ... Quiere decir que mientras dediquemos todas o casi todas nuestras posibilidades humanas, técnicas y materiales, y todos nuestros esfuerzos coordinados y estatales al cultivo único de exportación, y mantengamos en un rango de inferioridad y desarreglo social y económico los demás cultivos, todas las instituciones culturales de finalidad popular y toda la legislación de inspiración social, no lograrán efectivos resultados (Facio, 1972, pp. 124).

La expansión del mercado interno se basaría en la pequeña producción, porque se trataba de la forma mayoritaria de gestión productiva, en el marco de una economía predominantemente agrícola (Facio, 1972):

$\mathrm{Al}$ aumento y diversificación de la producción nacional, debe llegarse por el estímulo, la defensa y la organización de la pequeña propiedad (pp.169)

[...] las esperanzas inmediatas de mejoramiento material y moral para todos los costarricenses están en la organización y el incremento agrícolas: las del campesino, el peón, el pequeño propietario, el colono, el sin trabajo de los campos; y hasta las de los hombres de la ciudad: obreros, empleados, profesionales, artesanos, etc., que verán aumentar sus niveles de vida en su propia actividad, y abrirse nuevos campos donde aplicar su esfuerzo, ya sea en el campo, ya en la ciudad" (pp.173). 
Revista de Política Económica y Desarrollo Sostenible • EISSN: 2215-4167

Vol. 6 (1) • Julio-Diciembre, 2020: 1-24

DOI: https://doi.org/10.15359/peds.6-1.1

Esquivel

URL: http://www.revistas.una.ac.cr/politicaeconomica

Pero, el impulso del mercado interno no negaba la importancia de la exportación, acorde con las necesidades del desarrollo nacional. Se proponía que el mercado interno y el externo crecieran en armonía, para beneficio de la población nacional:

No se trata de terminar con el café ni aun de negarle el apoyo nacional - bien comprendemos que debe continuar siendo nuestra principal actividad económica -; sólo de emprender una campaña decidida para crearle al país nuevas fuentes de riqueza de mercado externo e interno, que al restarle a la del café su preeminencia absoluta [...] hagan posible material y espiritualmente que Costa Rica prosiga, o más bien, reanude su desarrollo de sociedad democrática y nación independiente. Conste claro: apoyaríamos, incluso, nuevas medidas de estímulo y protección para el café, siempre que fuesen ideadas como parte de un gran plan de reorganización y vigorización racionales y democráticas de la economía nacional (Facio, 1972, pp.106).

El nuevo modelo económico tendría mayor intervención del estado, dejando atrás el liberalismo típico de finales del siglo XIX e inicios del siglo XX. Se trataría de una economía capitalista donde la actividad privada se complementaba con las acciones y las regulaciones del Estado:

Todos estos principios son manifestaciones del criterio liberal constructivo moderno, según el cual, el Estado no debe ni desentenderse en un criminal laissez faire de los resultados de la economía, suponiéndolos automáticamente garantizados por su libre juego, ni intervenir arbitrariamente en su funcionamiento y sus resultados necesarios, ni arrogarse la dirección de la vida nacional entera, suponiendo en un gran plan totalitario la garantía de la felicidad colectiva [...]

El Estado liberal constructivo no atenta contra la propiedad privada; por el contrario busca, organizándola, hacerla más segura para el individuo y más útil para la comunidad; no pretende tampoco terminar con la libertad económica; su intervención, más bien, tiende, mediante un sistema de preventivos y limitaciones, a garantizarla (Facio, 1972, pp.171).

Asimismo, el modelo propuesto asignaba un papel a la inversión extranjera, de acuerdo con las necesidades de la expansión productiva nacional:

El objetivo a conseguir debe ser la explotación de los recursos nacionales con capital extranjero, en provecho nacional... No se persigue simplemente el rechazo del capital extranjero... sino más bien de multiplicar su intervención cuando lo demanden las urgencias de nuestro desarrollo, pero sujetándola al contralor estatal, para que su actividad implique, sin mengua del legítimo interés foráneo, verdadero acrecentamiento de la riqueza patria" (Facio, 1972, pp.175). 
Revista de Política Económica y Desarrollo Sostenible • EISSN: 2215-4167

Vol. 6 (1) • Julio-Diciembre, 2020: X-XX

DOI: https://doi.org/10.15359/peds.6-1.1

Esquivel

URL: http://www.revistas.una.ac.cr/politicaeconomica

En consecuencia, el pensamiento de Facio revelaba una clara definición del desarrollo como una combinación de la expansión del mercado interno con el crecimiento del mercado externo. De acuerdo con su visión, sería falsa la supuesta oposición entre mercado interno y externo. Además, en su estrategia, el crecimiento armónico de lo interno con lo externo estaba orientado a la satisfacción de las necesidades de la población: lo económico y lo social se implican mutuamente.

\section{2.- El resultado de la estrategia de desarrollo aplicada desde mediados del siglo $\mathrm{XX}$}

Los resultados políticos de las décadas del 40 y del 50 generaron el espacio institucional para que se aplicara en Costa Rica la concepción de un desarrollo productivo más amplio. No todo se puso en práctica, pero se hizo realidad lo básico del cambio en la estrategia de desarrollo. La producción agrícola se diversificó mediante el fortalecimiento de la agricultura que abastecía al mercado local. El monocultivo siguió siendo muy importante, pero acompañado de una agricultura local que adquirió más peso.

El café y el banano continuaron como los principales productos de exportación; incluso ampliaron la extensión cultivada, lo que se combinó con incrementos importantes de la productividad. La agricultura para el mercado local dispuso del estímulo suficiente para abastecer al consumidor nacional, jugando un papel más significativo en la estructura productiva del campo. Asimismo, la agroexportación experimentó cierta diversificación al surgir dos nuevos productos para la exportación: el azúcar y la carne. Estos nuevos productos aprovecharon nichos muy particulares del mercado internacional, aportando un complemento en la generación de ingresos externos para la economía.

El fortalecimiento del mercado local permitió el impulso de las industrias tradicionales que pudieron expandir sus volúmenes de producción. La rama tradicional de mayor peso era la producción de alimentos y bebidas, propiedad de un incipiente sector empresarial nacional. Luego se contaba con la rama de artículos para la vestimenta, menos desarrollada que la primera. Ambas ramas tomaron un impulso significativo con el crecimiento industrial de las décadas del 50 y del 60, lo que consolidó a ciertos grupos empresariales nacionales y permitió una participación creciente del capital extranjero. (Esquivel, 1985, pp.82, 91, 92)

De manera similar, surgieron nuevas ramas industriales que aportaron una mayor variedad de productos para el consumo de la población. En particular, la diversificación permitió el fortalecimiento de las ramas de producción química y metal mecánica, generando nuevos sectores empresariales y ampliando el grado de participación del capital extranjero. (Ibíd, pp.92, 93)

$\mathrm{Al}$ crecer la producción de manera diversificada se generaron diversos estímulos para que se expandieran las ramas de actividad complementaria, como el comercio, las finanzas, las comunicaciones, la recreación y similares. También, por el énfasis redistributivo que tenía el modelo, los servicios clásicos experimentaron un crecimiento considerable. Al respecto, el impulso a la educación y la salud jugó un papel esencial en la expansión de este tipo de servicios. 
Como resultado de esta transformación productiva, el Producto Interno Bruto (PIB) creció de manera acelerada durante las décadas del 50 y del 60. La ampliación del mercado interno generó un aumento del gasto realizado por los residentes en el país: consumo privado, inversión privada y gasto de gobierno. Una parte importante del gasto se destinó a la compra de productos locales, que eran suministrados por la agricultura dedicada al mercado interno y la industria que se expandía para atender las necesidades del demandante local.

El gráfico 1 muestra, mediante la línea continua, que la producción para el mercado local creció de manera acelerada, principalmente en la década del 60. De esta manera, la producción de las empresas que atendían el mercado local aumentó su tamaño en dos veces y medio, durante los años 50 y 60.

Gráfico 1 .

Costa Rica. Índice del crecimiento del producto según el mercado de destino $1952,1962,1972$

$1952=100$

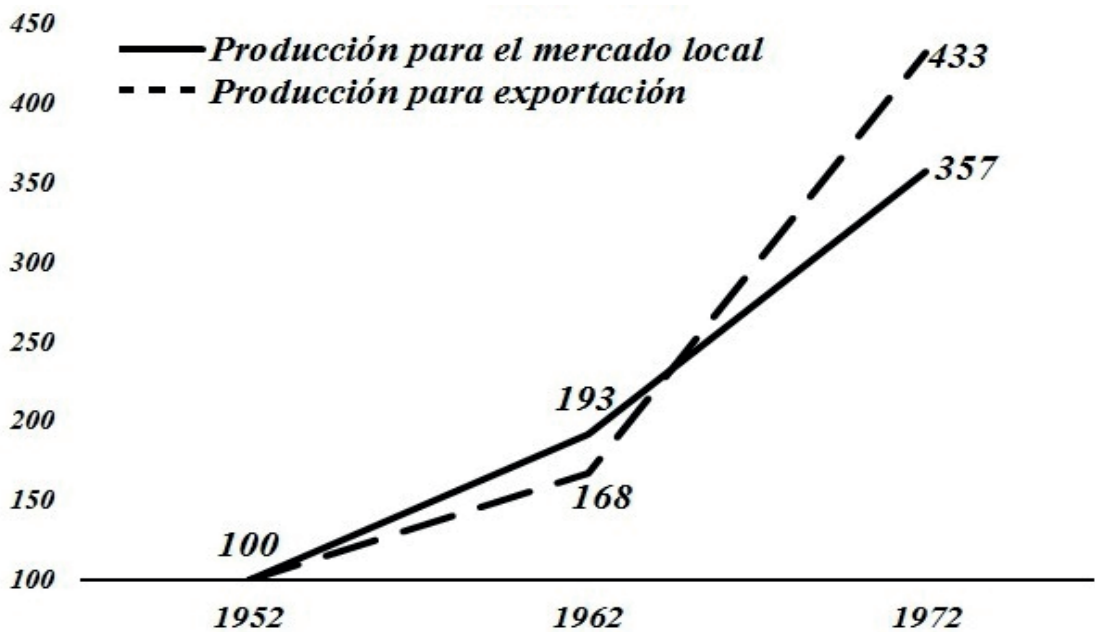

Fuente: Elaboración propia con datos del Banco Central de Costa Rica (1952, 1962, 1972)

La estrategia de desarrollo productivo aplicada en esa época creó las condiciones para que aumentara la demanda de los residentes, principalmente de los sectores más rezagados. Al respecto, se estableció una política de salarios crecientes que se orientaba al aumento de la capacidad de compra de las familias para estimular el consumo privado. En la misma dirección, se estimuló la inversión privada mediante diversos mecanismos, destacándose el papel del crédito, basado en la banca nacionalizada. Dado el tamaño pequeño del mercado interno, el mercado centroamericano suministró un impulso complementario mediante la puesta en 
Revista de Política Económica y Desarrollo Sostenible • EISSN: 2215-4167

Vol. 6 (1) • Julio-Diciembre, 2020: X-XX

DOI: https://doi.org/10.15359/peds.6-1.1

Esquivel

URL: http://www.revistas.una.ac.cr/politicaeconomica

marcha del Mercado Común Centroamericano que generaba una zona de comercio más amplia para la industria nacional, en la medida en que era posible vender a las pequeñas capas de clase media y alta que se encontraban en los países de la región centroamericana, principalmente en El Salvador y Guatemala. Desde luego, este último estímulo se contabilizaba como exportación.

Finalmente, se incrementó sustancialmente la capacidad de acción del Gobierno Central, (integrado por los ministerios del Poder Ejecutivo y por los otros poderes de la República) mediante el aumento de la carga tributaria. Se observó el fortalecimiento del impuesto sobre la renta y de los impuestos indirectos liderados por el impuesto sobre las ventas y una serie de impuestos específicos. De esta manera, el mecanismo impuesto - gasto público generó una redistribución que impulsó la expansión del gasto social, además de la inversión en infraestructura productiva. A lo anterior se agregó la conformación de una serie de instituciones autónomas que complementaban el accionar de los ministerios. Normalmente, el financiamiento de dichas instituciones significó la aplicación de una carga impositiva adicional al establecerse recargos específicos a las personas y a las empresas, de acuerdo con la naturaleza de cada entidad.

En términos macroeconómicos, dicho resultado fue completamente manejable, gracias al desempeño del sector exportador agrícola. En ese sentido, se expresó claramente el concepto de Rodrigo Facio en cuanto a la necesidad de una complementariedad entre el mercado interno y el sector exportador. El hecho de que una economía pequeña decida ampliar su desarrollo productivo, impulsando el mercado interno, no significa que se aísle del mundo. Por el contrario, debe revitalizar el vínculo con el exterior, para hacer viable el "desarrollo interno". Debe existir complementariedad entre el desarrollo hacia adentro y el desarrollo hacia afuera.

En consecuencia, durante las décadas del 50 y del 60, el proceso de expansión del mercado interno no limitó el crecimiento del sector exportador. Por el contrario, se estimuló la ampliación de las actividades tradicionales (café y banano) y se agregaron nuevos productos de exportación, incluyendo los productos industriales que salían hacia Centroamérica. El gráfico 1 reporta, a través de la línea discontinua, que el dinamismo de la exportación fue levemente menor al de la producción para el mercado local, durante la década del 50. No obstante; en la década del 60, la expansión exportadora fue notoria, mostrando un ritmo de crecimiento superior al de la producción para el demandante local. Así, el tamaño de la producción para exportación aumentó en más de tres veces, durante las décadas citadas.

Lo anterior revela que no existió contradicción entre el crecimiento del mercado interno y la expansión exportadora. Por el contrario, el incremento de las exportaciones, desde el punto de vista de la generación de ingresos externos, fue el gran complemento de la expansión industrial. Una parte importante del crecimiento de las importaciones obedecía a las necesidades del dinamismo industrial. De ahí que, al crecer las exportaciones agrícolas, el proceso exportador generó el financiamiento en dólares que se necesitaba para mantener el equilibrio de la balanza de pagos. 
Revista de Política Económica y Desarrollo Sostenible • EISSN: 2215-4167

Vol. 6 (1) • Julio-Diciembre, 2020: 1-24

DOI: https://doi.org/10.15359/peds.6-1.1

Esquivel

URL: http://www.revistas.una.ac.cr/politicaeconomica

\section{El límite de la estrategia aplicada a mediados del siglo XX y el resurgimiento del enfoque liberal-conservador}

La etapa de crecimiento resultante de la estrategia aplicada a mediados del siglo XX fue ampliamente estable en términos macro financieros. Las presiones de balanza de pagos que generaba el grado de apertura de la economía no se expresaron en problemas financieros, quedando controlada la dependencia de las importaciones que tenía la estructura productiva. Sin embargo; la estabilidad macro financiera se terminó en los años 70. A mediados de la década se produjo un primer aumento de precios del petróleo que cambió la historia de la economía mundial. Luego, al final del lapso, se experimentó un segundo aumento, que terminó de golpear drásticamente el largo período de expansión mundial, iniciado en la posguerra. En el país se sufrieron varios impactos, algunos directos y otros indirectos. Por una parte; el aumento del precio del petróleo afectó directamente el monto monetario de las importaciones. Comprando lo mismo, o menos, de petróleo, se pagó una cantidad mayor, en dólares. Por otra parte; el aumento del precio del petróleo afectó toda la economía mundial, generando una inflación generalizada. Así, el país observó que todo lo que compraba en el exterior, productos terminados o materias primas, tenía precios más altos. Además, el aumento del petróleo quebró el ritmo de crecimiento de las economías más desarrolladas, por lo que se produjeron diversos episodios de estancamiento o contracción de los mercados mundiales, afectando nuestras exportaciones.

Ante este panorama, la Balanza Comercial comenzó a mostrar déficits crecientes, a mediados de la década del 70. Al final de esos años, el modelo estaba prácticamente quebrado, en términos macro financieros. Se combinaron el grado de apertura de la economía con el deterioro del comercio internacional para generar un resultado insostenible de cuenta corriente (Balanza Comercial más las otras operaciones corrientes de la economía). La contracción de la economía mundial, la baja de los precios de exportación y el alza de los precios de importación, generaron menos ingresos y más gastos en la economía nacional. El déficit de cuenta corriente en 1980 fue superior a los US\$600 millones.

La década del 70 dejó claro que se acabó el margen externo, tal y como estaba planteado hasta ese momento. Por lo tanto, había que realimentar la dinámica económica con un nuevo esfuerzo exportador. Es decir; había que reorganizar la relación del desarrollo hacia afuera con el desarrollo hacia adentro, buscando preservar lo básico del legado: la expansión de la base material de la sociedad para mejorar el nivel de vida de todos. El proceso de desarrollo que se extendió de la década del 40 hasta la del 70 fue una forma determinada de combinar el desarrollo hacia afuera con el desarrollo hacia adentro. Luego de eso, no quedaba más opción que repensar esa relación y diseñar una nueva etapa, siempre marcada por ambos factores.

No obstante; el enfoque liberal-conservador dominó la reconstitución del proceso exportador. Durante la década del 80 las ideas liberales tenían mucha aceptación en el mundo. Los organismos financieros internacionales impulsaban esta forma de pensamiento económico, además de que centros académicos de relieve mundial tomaron el enfoque como el correcto para resolver los problemas de los países atrasados. También influyó en este predominio liberal la presencia de gobiernos conservadores en los principales países desarrollados. 
Revista de Política Económica y Desarrollo Sostenible • EISSN: 2215-4167

Vol. 6 (1) • Julio-Diciembre, 2020: X-XX

DOI: https://doi.org/10.15359/peds.6-1.1

Esquivel

URL: http://www.revistas.una.ac.cr/politicaeconomica

La base de la "victoria conceptual" del enfoque liberal-conservador fue un diagnóstico de las causas de la contracción productiva y de los desequilibrios macro financieros de la década del 80 que distorsionó la percepción de la realidad, pero que fue ampliamente aceptado. Asumiendo que el desarrollo de las décadas del 50, 60 y 70 había sido un período gobernado por la expansión del mercado interno, deprimiendo al sector exportador, se planteaba que debía olvidarse el papel del mercado interno y volver a la "esencia" de una economía pequeña que debía ser la exportación. Sin embargo; no es cierto que esa etapa deprimió la exportación; por el contrario, fue el fortalecimiento del sector exportador lo que generó el "margen económico", principalmente en lo externo, que hizo posible la expansión del mercado interno. Existió un funcionamiento articulado entre lo externo y lo interno, que permitió un mayor desarrollo nacional y una etapa de inclusión social. El resultado de esa etapa fue incompleto. No obstante; debe aceptarse que generó más desarrollo productivo, superando la obsoleta visión oligárquica de la necesaria exclusividad del proceso exportador.

Lo adecuado era plantearse cómo revitalizar el proceso exportador para mantener y ampliar lo alcanzado, mediante la reconstitución del mercado interno, sobre una nueva base de eficiencia económica y una profundización de los mecanismos de inclusión. Pero, aceptado el diagnóstico liberal-conservador, se hizo lo contrario. El diagnóstico distorsionado permitía desacreditar el papel del mercado interno, como el causante de la crisis, llamando a poner en su “justa dimensión” la acción del sector exportador.

En cierta forma resurgió el enfoque oligárquico que da importancia central a un crecimiento basado en la exportación sin articulación con la dinámica interna: maximizando la exportación, mediante la masificación de los espacios exportadores hasta el último metro cuadrado de la geografía nacional, recordando la expresión de Rodrigo Facio, se tendrían los ingresos para comprar "lo necesario", que no debía producirse en el país. ¿Cómo llegaban esos productos necesarios a los sectores amplios de la población? No se definía. De alguna forma habría goteo hacia todos. En esencia, resurgía el enfoque conservador de la oligarquía cafetalera: el desarrollo no es necesario, como articulación progresista de lo interno y lo externo, en el marco de un enfoque de inclusión. Lo importante era crecer mediante la exportación. El resto se vería luego.

Este enfoque avanzó en el manejo de la administración pública como tendencia dominante; lo que no niega la presencia de contra tendencias. O sea; la estrategia se ha aplicado a lo largo de las últimas tres décadas, dando como resultado un cambio sustancial en el patrón de crecimiento y en la distribución de sus frutos. Al inicio de este proceso de aplicaron las herramientas ortodoxas con el ingreso del país a la Organización Mundial del Comercio (OMC) y la ejecución de tres programas de ajuste estructural, en estrecha consonancia con el pensamiento del Banco Mundial, de ese momento.

Luego, el proceso continuó con acciones gubernamentales que consolidaron el proceso de apertura comercial sin la aplicación de una verdadera reconversión productiva que preparara al aparato productivo para la competencia externa. Sin embargo; existieron contra tendencias. Algunos gobiernos, o ministerios particulares, en algunos momentos expresaron posiciones social demócratas o social cristianas que se contrapusieron a la tendencia principal. Este no 
Revista de Política Económica y Desarrollo Sostenible • EISSN: 2215-4167

Vol. 6 (1) • Julio-Diciembre, 2020: 1-24

DOI: https://doi.org/10.15359/peds.6-1.1

Esquivel

URL: http://www.revistas.una.ac.cr/politicaeconomica

es el espacio para documentar estas contra tendencias, pero sí es importante dar cuenta de su existencia. En todo caso, la tendencia dominante predominó sobre las contra tendencias. Prueba de esto son los resultados macroeconómicos y sociales que se han producido, al acumular tres décadas de la nueva estrategia exportadora.

Desde luego, el riesgo de que no sucediera el derrame era muy amplio. En sentido estricto, el derrame fue parcial, como propagación de los frutos del crecimiento a todos los sectores de la población. Sin embargo; debe reconocerse que se duplicó el ingreso por habitante, desde inicios de la década del 90 hasta el fin de la década de 2010. No obstante; ese incremento del ingreso no llegó a todos. Se benefició el segmento de población, con mayor preparación técnica y profesional, que pudo insertarse en los sectores productivos basados en el ensamblaje. Debe mencionarse que se utilizaron los mecanismos de compensación asistencialista para amortiguar la insuficiencia del derrame, pero con resultados parciales, lo que se expresó en la persistencia del nivel de pobreza en el $20 \%$ de la población.

La expansión exportadora costarricense de fines del siglo XX y de inicios del siglo actual ha sido considerada, en algunos análisis, como un milagro de crecimiento. Se superó el esquema mono exportador, diversificando las líneas de producción para la exportación, incrementando notablemente los ingresos externos de la economía. Ese es un dato muy importante de la historia macroeconómica de las últimas décadas. El gráfico 2 muestra, a través de la línea discontinua, que la producción para la exportación aumentó casi tres veces, durante las décadas de 90 y de 2000; algo similar a lo visto en el gráfico 1. Este fue el principal estímulo para que la economía creciera durante las últimas décadas. Pero, reducir el análisis al simple hecho de la exportación es limitado, sin considerar los otros aspectos del crecimiento productivo y el perfil macro financiero que se generó durante esta etapa.

Gráfico 2.

Costa Rica. Índice del crecimiento del producto según el mercado de destino $1992,2002,2012$

$1992=100$

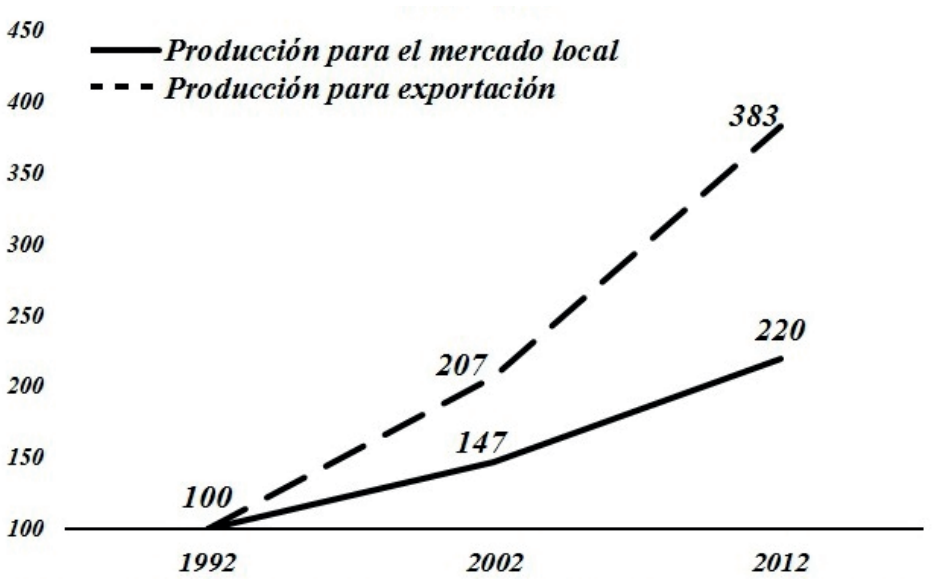

Fuente: Elaboración propia con datos del Banco Central de Costa Rica (1992, 2002, 2012) 
La estrategia que impulsó el nuevo crecimiento exportador no tenía interés en la producción para el mercado local. Lo que se buscaba era el crecimiento, basado en la exportación, sin importar los efectos dinámicos sobre amplios sectores productivos nacionales, en particular las pequeñas y medianas empresas. En el gráfico 2 se observa, a través de la línea continua, que la producción para el mercado local creció de manera más lenta, comparando con el crecimiento de la exportación. En ese sentido, existió un divorcio entre la expansión exportadora y el dinamismo de la producción que se orientaba a las necesidades del comprador local. Si se comparan los gráficos 1 y 2 , es posible apreciar las diferencias de articulación de lo externo con lo interno, en las dos estrategias de desarrollo citadas.

De esta manera, la expansión productiva no generó encadenamientos relevantes. Desde luego, hay ciertos tipos de producción agrícola que se usaron para desarrollar producto exportable que escapa a esta observación. Sin embargo; el sector agrícola, aunque exportó, nunca fue una prioridad de la estrategia de crecimiento. En este contexto, la expansión industrial para exportación se ha desarrollado como un nuevo proceso de ensamblaje que no genera encadenamientos relevantes, hacia el conjunto de la estructura productiva. El gráfico 3 muestra la capacidad limitada que tiene el sector dinámico del nuevo modelo exportador para difundir su expansión hacia el resto de la economía. Tal gráfico reporta los multiplicadores hacia atrás de la mayor parte del aparato productivo (98 \% del PIB). Este indicador mide la cantidad de colones que genera el resto de la economía por cada 100 colones producidos por un sector productivo.

Las áreas de la producción orientadas al uso de los recursos materiales propios del país (la agricultura, la agroindustria, el turismo y otros similares), que generan el $35 \%$ del PIB, reportan los multiplicadores hacia atrás más elevados de la economía. Por el contrario, las áreas de producción que se basan en el ensamblaje, con una participación del $15 \%$ en el PIB, producen un efecto multiplicativo que es la mitad de lo que hacen los sectores tradicionales. La industria de primer ensamblaje corresponde a la producción establecida para el mercado local y el Mercado Común Centroamericano hace seis o siete décadas. La industria de segundo ensamblaje reporta la nueva producción industrial y de servicios de zona franca de las últimas décadas. Los datos del gráfico son promedios por sector. Si se desagregan las diferencias son más llamativas. Por ejemplo, la elaboración de embutidos (de diferentes carnes) produce un multiplicador hacia atrás de 116, mientras la producción de equipo médico (la joya de la corona) genera un multiplicador de 27.

Los servicios de apoyo, generados en zona franca, como la gestión de procesos contables y otros similares reportan un multiplicador de 31. Si un producto material, como el equipo médico debe traer del exterior la mayor parte de sus materias primas e insumos, necesariamente multiplica poco. Algo similar sucede con los servicios de zona franca porque son intensivos en recursos humanos, generando pocas compras al resto de la economía. 


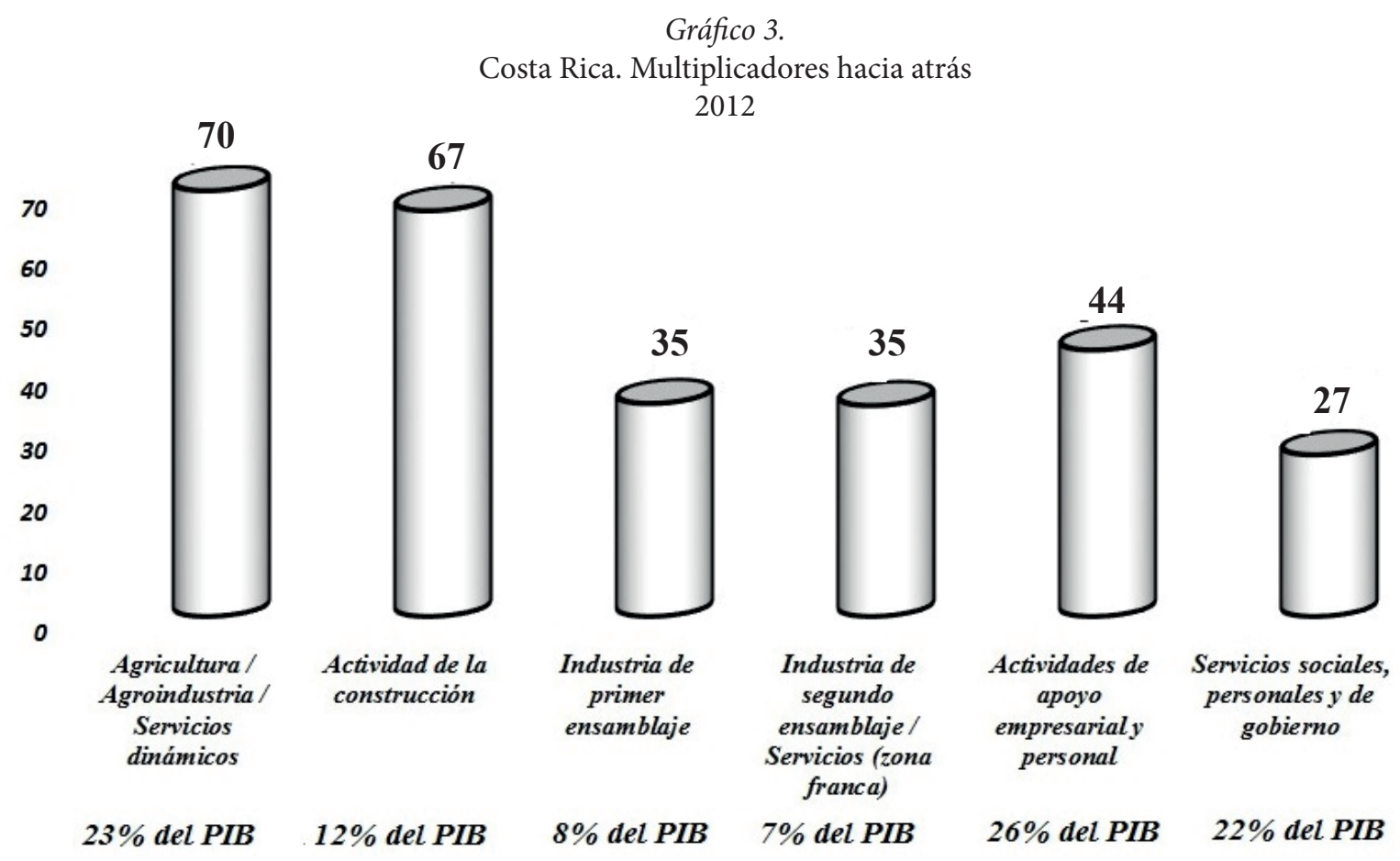

Fuente: Elaboración propia con datos del Banco Central de Costa Rica (2012)

Reconocer lo anterior, no significa ocultar el hecho de que tales sectores generan expansión. Lo que se destaca con este reconocimiento es lo limitado del tipo de expansión. Lo delicado de este tipo de apertura de la producción, basada en los procesos de ensamblaje, es que limita considerablemente las posibilidades de desarrollo de un país. La producción de ensamblaje normalmente obedece a la acción de una empresa externa al país, que busca reducir costos respecto al lugar original donde se elabora el producto o el servicio. Al respecto, las formas más típicas de reducción de costos, al trasladar partes de los procesos productivos a otro país, son el pago de salarios menores y la reducción del pago de impuestos. En el caso de Costa Rica, los salarios son más altos que los de la mayor parte de Latinoamérica; pero, son más bajos que los que se pagan en economías más avanzadas. Por lo tanto, relocalizar un espacio de producción en el país, mediante un proceso de ensamblaje, genera una ventaja de costo para la empresa que lo haga.

Pero, aceptar esto significa limitar las posibilidades de expandir la demanda orientada al mercado interno (a través del ingreso de la fuerza laboral) y condenar a las finanzas públicas a una permanente anemia de ingresos tributarios, porque el sector dinámico de la exportación no paga, o paga pocos impuestos. Estos aspectos son trabas fundamentales al desarrollo del país.

En el gráfico 3, también se muestran los multiplicadores hacia atrás de las áreas de apoyo y de los sectores sociales, que no son los espacios de producción que lideran el crecimiento, pero que 
tienen un tamaño importante (la mitad de la economía): Las áreas de apoyo contienen al comercio, el transporte, las finanzas y otros similares. Los sectores sociales están integrados por la salud, la educación, los servicios gubernamentales y otros afines. La capacidad multiplicativa de estos sectores es limitada porque la mayor parte son servicios que son intensivos en recursos humanos.

René Villarreal y Rocío Ramos, economistas mexicanos, han estudiado el papel de los procesos de ensamblaje en la nueva estrategia exportadora, llegando a conclusiones importantes, desde el punto de vista de estrategia de desarrollo. Al respecto, Villarreal y Ramos formulan lo siguiente, a partir de la experiencia mexicana, considerando que es válido para muchos de los procesos exportadores latinoamericanos (Villarreal y Ramos, 2001):

El modelo industrial exportador se basó en tres pilares fundamentales: 1) la apertura comercial y financiera y la inversión extranjera; 2) la liberalización de los mercados internos, y 3) una política de fomento industrial pasiva: "la mejor política industrial es la que no existe" [...] En los años noventa el modelo industrial exportador fue exitoso, en tanto que desarrolló una locomotora dinámica exportadora en México y algunos países de la región, como Chile. Actualmente esa locomotora presenta limitaciones importantes que habrá que corregir en el futuro. [...] En lo fundamental, la locomotora exportadora tiene un motor dinámico de crecimiento pero con bajo poder de arrastre. (pp. 777)

¿Cuáles son los efectos palpables de este desarrollo primario del modelo exportador?

1) La desarticulación de cadenas productivas... Es decir, el desempeño exportador no incide en el fortalecimiento del aparato productivo interno. Por ello la industria maquiladora tiene poco arrastre y por sí sola será incapaz de generar los empleos necesarios para un crecimiento dinámico y sustentable en las próximas décadas [...]

2) La concentración de las ventas externas [...]

3) Baja participación tributaria... puesto que las importaciones de maquila están exentas de aranceles y el resto paga aranceles bajos, el crecimiento hacia afuera basado en el comercio exterior puede ser dinámico pero no permite elevar el coeficiente tributario. (pp. 777-778)

\section{4.- Los desequilibrios de la estrategia liberal de exportaciones}

El aporte de Rodrigo Facio debe ubicarse en el contexto de la realidad costarricense de mediados de la década del 50 del siglo pasado. Jugó un papel en aquella época y su producción intelectual debe entenderse como un ingrediente fundamental que colaboró con la transformación del país y a la construcción de la Costa Rica de la segunda mitad del siglo pasado. Por lo tanto, no podemos tomar su obra para repetirla dogmáticamente como si fuera una receta universal que le sirve al país en cualquier momento. Lo que se debe rescatar es el concepto de desarrollo de una economía 
Revista de Política Económica y Desarrollo Sostenible • EISSN: 2215-4167

Vol. 6 (1) • Julio-Diciembre, 2020: 1-24

DOI: https://doi.org/10.15359/peds.6-1.1

Esquivel

URL: http://www.revistas.una.ac.cr/politicaeconomica

como la nuestra, independientemente de las especificidades del momento que vivió Facio. En ese sentido, lo central es observar la claridad con que definió el problema del desarrollo: se trata de buscar una combinación de la expansión del mercado interno con el crecimiento del mercado externo. De acuerdo con su visión, sería falsa la supuesta oposición entre el mercado interno y el externo. Además, en su estrategia, el crecimiento armónico de lo interno con lo externo estaba orientado a la satisfacción de las necesidades de la población: lo económico y lo social se implican mutuamente.

¿Por qué es importante hoy ese concepto? Porque el enfoque neoliberal renunció a la articulación progresista de lo interno con lo externo, en el nuevo proceso exportador de las últimas tres décadas. La expansión generada desde la mitad de la década del 80 del siglo anterior generó un crecimiento apreciable, pero basado en una macroeconomía desequilibrada. En primer lugar, se logró una expansión exportadora significativa pero incompleta, ya que se desaprovecharon oportunidades de crecimiento hacia el exterior, en sectores como la agroindustria, el turismo que genera encadenamientos y la producción de servicios de alto valor agregado. Estos sectores crecieron, pero no recibieron el respaldo apropiado de la estrategia gubernamental.

Asimismo, la expansión exportadora no se acompañó de una revitalización del mercado interno, que fue relegado a un papel de menor importancia como nicho de crecimiento del comercio importador. Como consecuencia, el desempleo se ha incrementado constantemente hasta llegar a afectar una quinta parte de la población, como se observa en el gráfico 4. La tasa de desempleo abierto (mostrado mediante una línea discontinua) ha subido en las últimas tres décadas. Algo similar ha sucedido con el subempleo, lo que genera, sumando ambos aspectos del desempleo, una tasa de subutilización (dibujado mediante una línea continua) que llega al $20 \%$ en los últimos años (2018 y 2019).

Gráfico 4.

Costa Rica. Desempleo abierto y subutilización total de la fuerza de trabajo

1992-2019

(en porcentajes)

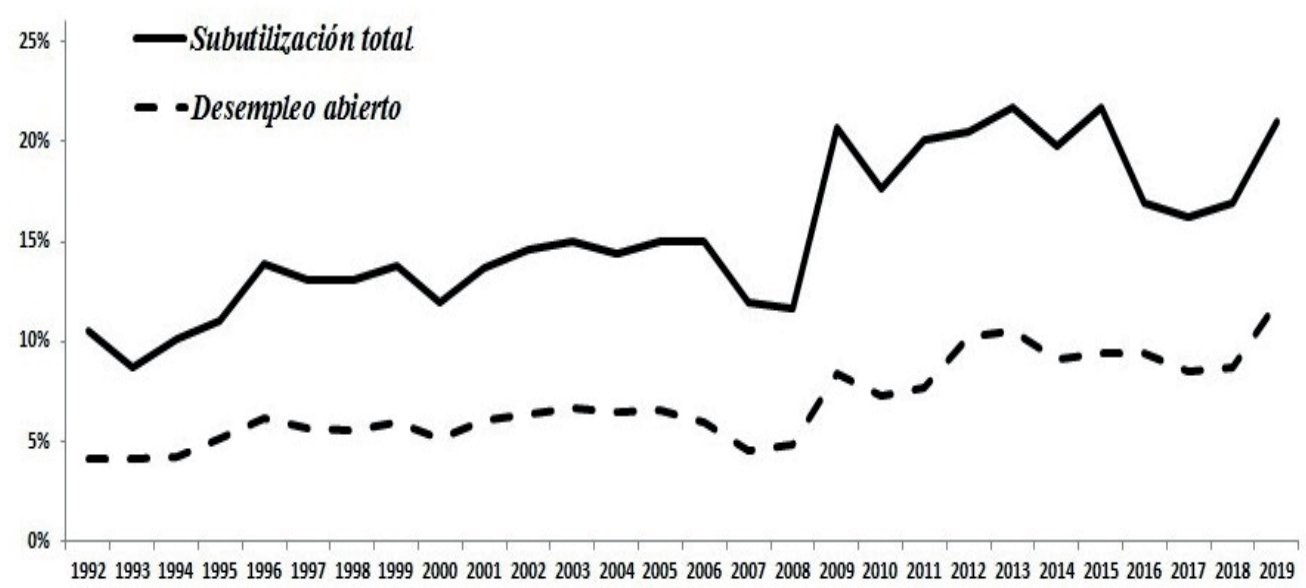

Fuente: Elaboración propia con datos del Instituto Nacional de Estadísticas y Censos (1992 - 2019) 
Además, el aumento del desempleo ha sido marcado por la exclusión. El gráfico 5 muestra claramente que los principales sectores excluidos del crecimiento han sido los quintiles I y II (donde se ubica la población con menores ingresos y de menor nivel educativo), ya que el desempleo abierto afecta principalmente a esos grupos de la población.

Gráfico 5.

Costa Rica. Desempleo abierto en los quintiles I,II y V

1998-2019

(en porcentajes)

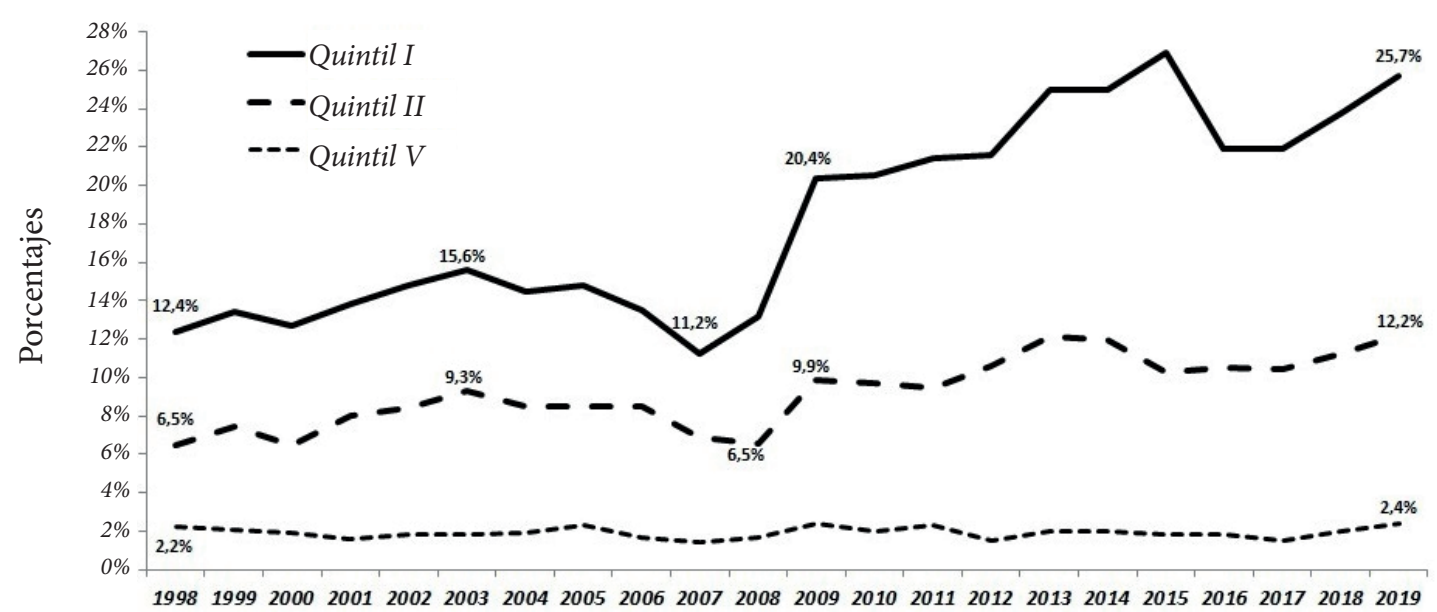

Fuente: Elaboración propia con datos del Instituto Nacional de Estadísticas y Censos (1998 - 2019)

En segundo lugar, el crecimiento se combinó con una apertura amplia del mercado interno y de la producción, por lo que en la cuenta corriente de la Balanza de Pagos se generó una quiebra constante, equivalente al $3 \%$ - $4 \%$ del PIB. El gráfico 6 muestra el resultado de esta Balanza de 2002 al 2019. La cuenta corriente (que se representa mediante cilindros negros) fue deficitaria todos los años. Aunque se produjo el milagro exportador, el incremento de los ingresos en dólares se gastó en un incremento de las importaciones que fue la consecuencia de la apertura acelerada, impulsada por el dogma liberal que recitaba la "importancia" del comercio internacional libre. Más allá de las compras necesarias para la producción, la importación de productos para el consumo, cada vez más libres de aranceles, y el alto nivel de importaciones para los procesos de ensamblaje, alimentaron este proceso de crecimiento acelerado de las compras al exterior.

La apertura de las últimas tres décadas no generó superávit (lo que postulaba el credo neoliberal) sino que produjo lo contrario: un déficit crónico. Esta característica no se manifestó en un desequilibrio externo gracias al comportamiento de los flujos de financiamiento provenientes del exterior, principalmente de la inversión extranjera directa. El gráfico en mención revela que el ingreso 
de recursos externos (dibujado con cilindros blancos) financió el déficit de la cuenta corriente. Incluso, el nivel del flujo de capital externo fue de tal magnitud que superó al déficit de cuenta corriente, por lo que se generaron incrementos de reservas recurrentes que engrosaron las arcas del Banco Central de Costa Rica (BCCR). Esto produjo un excedente estructural de dólares. Al iniciar la década del 2020 las reservas internacionales fluctúan alrededor de los US $\$ 8.000$ millones, lo que significa seis meses de importación, revelando una gran solvencia financiera de las reservas.

Finalmente, la economía logró una reducción apreciable de la inflación, hasta alcanzar un nivel de incremento de precios que se ha estabilizado en valores cercanos al $1 \%-2 \%$ anual, o, incluso, en algunos momentos se ha eliminado la inflación. Pero dicha estabilidad se sustentó en el excedente de dólares de la Balanza de Pagos, generado por el financiamiento externo, lo que eliminó la principal presión de inflación por empuje de costos, que es el comportamiento del tipo de cambio. Si en algún momento se perdiera el excedente de dólares, la estabilidad de precios se desplomaría por el efecto en el tipo de cambio, ante la falta de divisas para el mercado cambiario.

Gráfico 6.

Costa Rica. Balanza de pagos

2012-2019

(En millones de US \$)

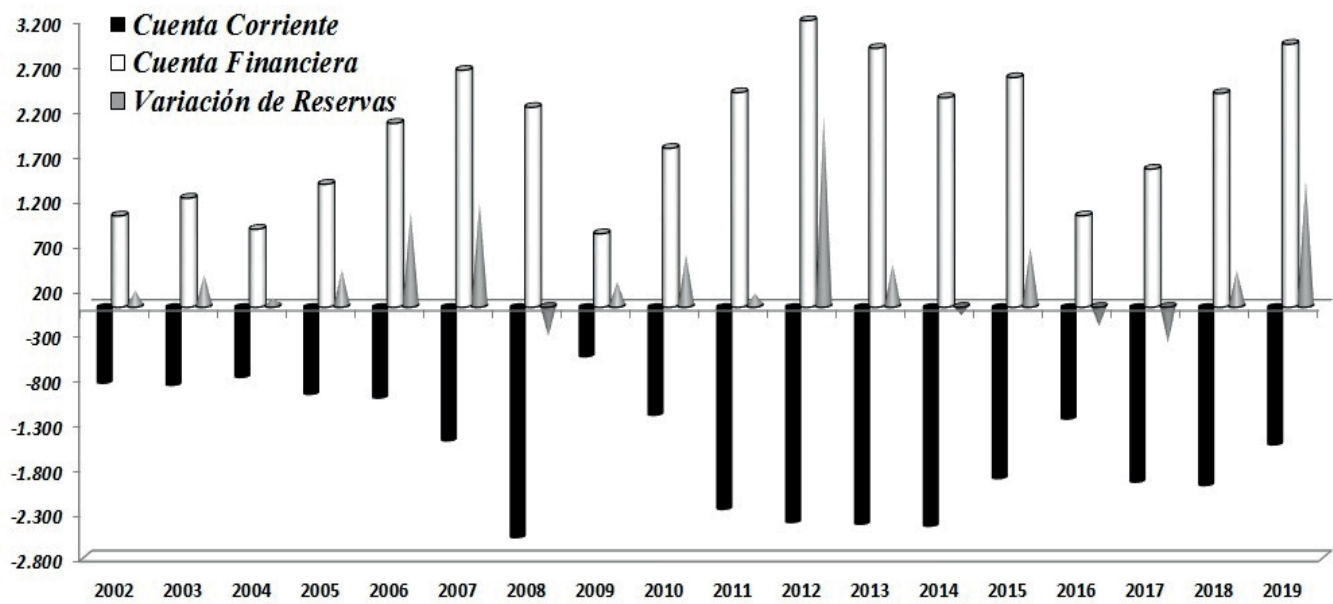

Fuente: Elaboración propia con datos del Banco Central de Costa Rica (2002 - 2019) 
Revista de Política Económica y Desarrollo Sostenible • EISSN: 2215-4167

Vol. 6 (1) • Julio-Diciembre, 2020: X-XX

DOI: https://doi.org/10.15359/peds.6-1.1

Esquivel

URL: http://www.revistas.una.ac.cr/politicaeconomica

\section{5.- Los desequilibrios actuales del desarrollo y la vigencia de Rodrigo Facio}

Un sistema económico que no puede brindar ganancias a la mayoría de sus ciudadanos, y en el cual una proporción creciente de la población se enfrenta a una inseguridad cada vez mayor, es, de manera fundamental, un sistema económico fracasado.

Joseph Stiglitz (2014)

La acumulación de malas experiencias aplicando estrategias de apertura orientadas por el enfoque neoliberal, y el desarrollo de procesos políticos nacionales que clamaron por la necesidad de un cambio de enfoque económico, que permitiera un verdadero desarrollo nacional, han propiciado una discusión más amplia sobre estrategias de desarrollo en las últimas dos décadas. En ese sentido, el concepto de preparación para el desarrollo conduce a la noción de la ventaja competitiva como resultado de un proceso en el que se busca resolver los rezagos de una estructura heterogénea, en la que hay individuos avanzados e individuos rezagados.

Resolviendo los rezagos se mejora la capacidad competitiva de los países y se propicia el crecimiento con inclusión, de ahí que con este concepto aparece una forma moderna de comprender la relación de lo externo con lo interno. Para el neoliberalismo todos los individuos son iguales, y la ventaja competitiva es natural, ya está adquirida antes de entrar al comercio internacional. Lo único que se debe hacer es poner a los individuos, que supuestamente son iguales y capaces -instantáneamente-, a "competir" en un mercado libre. Para un enfoque de preparación es lo opuesto: la ventaja competitiva se construye. Desde luego, tal construcción aprovecha las condiciones naturales y la experiencia productiva de cada país. Ricardo FfrenchDavis, economista chileno, lo formula de la siguiente manera (Ffrench-Davis 1999):

El neoliberalismo tiene una fe extrema en la eficiencia del sector privado tradicional y una desconfianza también extrema en el sector público y en las formas no tradicionales de organización privada. Existe una tendencia a efectuar reformas de manera abrupta y extrema, dando por supuesto que los mercados se tornan completos espontáneamente cuando se liberalizan [...] (pp. 4)

La diferencia analítica sustancial entre los enfoques neoliberal y neoestructuralista puede resumirse en los supuestos específicos sobre el grado de integración o segmentación de los mercados, y de homogeneidad o heterogeneidad de productos y factores. Esas diferencias en los supuestos de cada enfoque conducen a políticas divergentes dentro de un sistema de economía de mercado común a ambas alternativas, con implicaciones significativas en lo que respecta a la dinámica que adopta la distribución del ingreso, así como la asignación y generación de recursos productivos[...] (pp. 19) 
Revista de Política Económica y Desarrollo Sostenible • EISSN: 2215-4167

Vol. 6 (1) • Julio-Diciembre, 2020: 1-24

DOI: https://doi.org/10.15359/peds.6-1.1

Esquivel

URL: http://www.revistas.una.ac.cr/politicaeconomica

Es indispensable que la apertura de la economía se haga de tal modo que.... permita una efectiva reconversión de las actividades productivas. Una condición crucial es la gradualidad del proceso, asociada a la capacidad de respuesta de los diversos agentes económicos frente a los cambios inducidos por políticas públicas (pp. 39) ${ }^{3}$

De esta forma, con base en una noción como la planteada por Ffrench Davis, se ha desarrollado durante las últimas dos décadas una propuesta que acepta el fracaso de las políticas de choque, pero que plantea la necesidad de participar activamente en el comercio internacional, mediante una estrategia de gestión de ventajas competitivas sustentada en la generación de innovaciones, la profundización del aprendizaje y en la inclusión social. En consecuencia, se necesita un proceso de preparación que permita el cambio de las empresas y de su entorno sectorial y nacional.

$\mathrm{Al}$ respecto, se necesitan políticas educativas, políticas de desarrollo de la infraestructura y de la tecnología, entre otras, de acuerdo con los conceptos de Ffrench Davis. En consecuencia, la competitividad "auténtica", basada en la preparación, es un proceso de articulación de lo externo con lo interno. Así, el nuevo enfoque agrega un aspecto central a la estrategia de desarrollo, que no está en la visión simplista del neoliberalismo, como lo es la necesidad de efectuar un proceso de gestión de las ventajas competitivas. En el enfoque neoliberal, esto no está presente porque se asume que la ventaja competitiva es natural, ya existe al momento de decretar la apertura y opera de manera inmediata. La gestión de ventajas competitivas requiere de mucho tiempo, por lo tanto, se trata de un proceso gradual y selectivo.

La estrategia neoliberal, en esencia, es anti estatista. El concepto básico del enfoque neoliberal es la libertad de acción del individuo económico, ya sean empresas o consumidores. Para la visión liberal, si se logra que los individuos económicos participen libremente en los mercados, se producirá el mejor resultado posible: los empresarios maximizarán la producción, que será ofrecida a un precio acorde con la eficiencia productiva y con la mejor calidad disponible, mientras que los consumidores exigirán y obtendrán un producto de calidad al mejor precio factible en el mercado. En ese contexto, para la visión liberal el Estado es un obstáculo para el desarrollo. Si el libre funcionamiento de los individuos económicos produce el mejor resultado posible, ¿para qué queremos que el Estado intervenga en la economía? Por el contrario, la intervención del Estado, según este enfoque, producirá una distorsión en el funcionamiento de los mercados porque no dejará que los individuos actúen libremente, lo que se traducirá en un resultado no óptimo del mercado.

Tal enfoque anti estatista generó un daño importante a las funciones estatales en las últimas tres décadas. Se desmantelaron algunas funciones y otras quedaron reducidas a un nivel poco operativo. Todo esto, en un contexto de deterioro de la carga tributaria efectiva. Aunque nominalmente la carga impositiva es alta, no se cobra adecuadamente a los segmentos de mayor ingreso, recayendo el peso tributario en los grupos medios de la población. En particular, se observa un debilitamiento del aporte de las empresas de mayor tamaño al impuesto sobre la renta.

$3 \quad$ El texto destacado es del autor. 
Revista de Política Económica y Desarrollo Sostenible • EISSN: 2215-4167

Vol. 6 (1) • Julio-Diciembre, 2020: X-XX

DOI: https://doi.org/10.15359/peds.6-1.1

Esquivel

URL: http://www.revistas.una.ac.cr/politicaeconomica

Por el contrario, en una estrategia alternativa el Estado juega un papel importante. Pero no como un fin en sí mismo, sino como un elemento de apoyo que participa para que el individuo logre los objetivos de desarrollo. Para esto, el Estado debe ser inversionista y regulador, en el marco de una gestión eficiente que garantice el uso óptimo de los recursos públicos. El tamaño que resulte de esta intervención depende de la especificidad de la estrategia de cada país. No importa si el tamaño de la intervención es grande o pequeña. Lo esencial es que la intervención sea eficiente, pertinente y equilibrada financieramente.

Por lo tanto, una estrategia alternativa que busque la articulación entre lo externo y lo interno necesita reconstituir el funcionamiento del Estado. En ese sentido, debe recuperarse su capacidad de acción mediante el cobro de una carga tributaria apropiada, garantizando el aporte de todos los sectores de acuerdo con su nivel de ingreso. Es necesario relanzar el mecanismo impuesto - gasto público para propiciar la redistribución impulsando la expansión del gasto social, además de la inversión en infraestructura productiva.

En particular, el incremento del gasto social, debe ser una palanca de fortalecimiento del mercado interno, a través del estímulo del consumo de los segmentos más rezagados. Desde luego, se trata de impulsar un gasto social de calidad, mediante el uso óptimo de los recursos. En el caso de Costa Rica, esto pasa por la reconstrucción de los mecanismos de utilización de los recursos asignados al área social, principalmente al Fondo de Desarrollo y Asignaciones Familiares (FODESAF). Tal Institución recauda el $2 \%$ del PIB, pero el impacto de los programas que financia es limitado, lo que se comprueba con la permanencia de la pobreza en un nivel equivalente al $20 \%$ de la población.

En consecuencia, considerando que ya pasó el tiempo del neoliberalismo, es la hora de repensar la inserción al mercado internacional de las economías latinoamericanas. Reconociendo que muchos procesos de exportación, de las últimas décadas, generaron una expansión incompleta, desequilibrada, que margina al mercado interno, que genera exclusión, estamos frente al reto de impulsar una nueva etapa de exportación, orientada por nuevos conceptos, como lo indicado aquí.

De esta manera, la discusión estratégica actual se ubica en el mismo concepto de desarrollo de Facio. El reto está en formular una estrategia que enfrente el problema básico de la expansión con inclusión: el crecimiento armónico de lo interno con lo externo orientado a la satisfacción de las necesidades de la población. Para Facio, toda etapa de inserción en la economía mundial requiere la articulación de lo externo con lo interno para que impulse el desarrollo.

La tarea del presente enfrenta esa exigencia. No basta con el impulso de una exportación de ensamblaje, usualmente asociada al desmantelamiento de las funciones estatales y a la concentración del ingreso. La exportación relevante, desde el punto de vista del desarrollo, debe generar vínculos entre lo exportado y la dinámica del mercado local, en el marco de una acción estatal eficiente y pertinente, propiciando la expansión del empleo y la inclusión de amplios sectores de la población. Rodrigo Facio sigue vigente, aunque los términos instrumentales han cambiado de acuerdo con la especificidad del momento. 
Revista de Política Económica y Desarrollo Sostenible • EISSN: 2215-4167

Vol. 6 (1) • Julio-Diciembre, 2020: 1-24

DOI: https://doi.org/10.15359/peds.6-1.1

URL: http://www.revistas.una.ac.cr/politicaeconomica

\section{Referencias bibliográficas}

Esquivel Villegas, F. (1985). El desarrollo del capital en la industria de Costa Rica 1950 - 1970. Heredia, Costa Rica: Editorial de la Universidad Nacional.

Esquivel Villegas, F. (2015). Análisis macroeconómico de la estrategia de desarrollo costarricense. Un modelo desequilibrado que ha subsistido. San José, Costa Rica

Facio, R. (1972). Estudio sobre economía costarricense. En Obras de Rodrigo Facio Tomo I San José, Costa Rica: Editorial Costa Rica.

Ffrench-Davis, R. (1999). Macroeconomía, comercio y finanzas: para reformar las reformas en América Latina. McGraw Hill.

Stiglitz, J. (octubre, 2014). La edad de la vulnerabilidad. Project Syndicate.

Villarreal, R., \& Ramos, R. (2001). La apertura de México y la paradoja de la competitividad: hacia un modelo de competitividad sistémica. Comercio Exterior, 51(9), 772-788. 